\title{
Accertamento e scelta dei dispositivi ad accesso venoso
}

\author{
Assessment and choice of the device for vascular access
}

\author{
Stefano Finotto ${ }^{1} \quad$ Roberto Caroli $^{2} \quad$ Maura Cabrini $^{3} \quad$ Simona losi $^{4} \quad$ Daniela Mecugni $^{5}$
}

\begin{abstract}
RIASSUNTO
L'esecuzione da parte degli infermieri di un accurato accertamento vascolare all'inizio e per tutta la durata della terapia infusiva, insieme all'individuazione del dispositivo più adatto al paziente, può aumentare la soddisfazione del paziente, diminuire le complicanze, preservare le vene periferiche, diminuire o eliminare il tempo impiegato dagli infermieri a reperire accessi venosi improbabili, ridurre i costi associati alla terapia infusiva.

Gli obiettivi sono stati di indagare la presenza di una procedura per la scelta del dispositivo venoso più adeguato al paziente, descrivere i criteri utilizzati per la valutazione del letto vascolare, identificare il momento in cui gli infermieri eseguono la valutazione del letto vascolare.

E' stato svolto uno studio descrittivo con campione di convenienza composto da 290 infermieri. Lo strumento di raccolta dati utilizzato è un questionario anonimo. I questionari somministrati, restituiti e ritenuti validi sono stati 227. Il $14 \%$ del campione afferma che è in uso una procedura per valutare il dispositivo venoso più adatto per ogni paziente mentre, il $70 \%$ dichiara che non è in uso e il $16 \%$ che non sa se c'è una procedura.

I risultati dello studio mostrano che nelle unità operative appartenenti al campione (1) non è in uso una procedura per la scelta del dispositivo più adeguato al paziente, (2) che gli infermieri non hanno chiari i criteri per effettuare la valutazione del letto vascolare, (3) che essi effettuano la valutazione del letto vascolare in momenti differenti del processo infusivo.
\end{abstract}

Parole Chiave: accertamento vascolare, selezione dispositivo venoso, accesso vascolare, terapia infusiva, linee guida pratiche

ABSTRACT

The accurate vascular monitoring by nurses both at the beginning and during infusion treatment, in addition to the selection of the best device for each patient, can increase patient satisfaction, reduce complications, preserve peripheral veins, reduce or eliminate time used to find unlikely vein access, reduce hospital stay length and reduce the costs associated with infusion treatment.

Aims of study are to ascertain the presence of a procedure for choosing the most suitable venous device for each patient, to identify nurses' criteria to make a vascular assessment, and to describe when nurses make a vascular assessment.

A descriptive study with convenience sample of 290 nurses. The data collection instrument is an anonymous questionnaire. The questionnaires administered, returned, and considered valid were $227.14 \%$ of the sample states that within their ward there is a a procedure to evaluate the most suitable device, while $70 \%$ states the opposite and $16 \%$ of the sample states that "they do not know".

The results show fairly clearly that in the wards considered there is no procedure currently in use in order to choose the most suitable device for each patient, that nurses do not have clear criteria to make a vascular assessment, that they carry out vascular assessment at different times of the infusion's process.

Key words: vascular assessment, venous device selection, vascular access, infusion therapy, practice guidelines

\section{INTRODUZIONE}

$I^{\mathrm{din}} \mathrm{s}$ dispositivi endovenosi sono un importante e comune aspetto della pratica ospedaliera per la somministrazione di farmaci, sostanze nutritive, fluidi, prodotti del sangue e di controllo dello status emodinamico del paziente. Negli Stati Uniti sono usati annualmente circa 150 milioni di cateteri intravenosi la maggior parte dei quali sono cateteri periferici (Lundgren et al., 1996). Tuttavia, i dispositivi intravascolari possono provocare

1 Dott. Mag. in Scienze Infermieristiche e Ostetriche, Tutor Corso di Laurea in Infermieristica Università degli Studi di Modena e Reggio Emilia, sede di Reggio Emilia.

2 Coordinatore Infermieristico, Azienda Ospedaliera di Reggio Emilia

3 Infermiera, Azienda Ospedaliera di Reggio Emilia

4 Infermiera, Azienda Ospedaliera di Reggio Emilia

5 Ricercatore MED/45, Università di Modena e Reggio Emilia una serie di complicanze che aumentano la morbosità e la lunghezza dell'ospedalizzazione (Kagel et al., 2004). L'incidenza delle infezioni del sangue catetere-venosoperiferico-correlate è stimata allo 0.5 per 1000 giornicatetere (Maki et al., 2006). La complicanza più comune legata ai dispositivi venosi nei pazienti adulti è la tromboflebite, che si sviluppa nel 4 - 45\% dei pazienti e che ha un'incidenza media del 30\% (Tagalakis et al., 2002; Myrianthefs et al., 2005). Lo sviluppo della tromboflebite è legato alle condizioni fisiche del paziente, all'effetto tossico delle infusioni somministrate e alla gestione del catetere venoso periferico, in particolare al tempo di permanenza, al materiale di composizione del dispositivo, al sito di inserzione, al calibro, al metodo di fissaggio, al tipo di medicazione 
e alla qualità della documentazione e della registrazione (Maki et al., 1987; Smith, 2006).

Gli infermieri hanno importanti responsabilità nell'applicazione e gestione dei cateteri venosi. Essi inseriscono i dispositivi, monitorano gli effetti terapeutici dei fluidi infusi e la comparsa di eventuali complicanze. Un accurato accertamento vascolare all'inizio e per tutta la durata della terapia infusiva, insieme all'individuazione del dispositivo più adatto, possono aumentare la soddisfazione del paziente, diminuire i ritardi di infusione della terapia legati alla perdita dell'accesso venoso, diminuire le complicanze, preservare le vene periferiche, diminuire il tempo impiegato dagli infermieri a reperire accessi venosi, diminuire la durata dell'ospedalizzazione, diminuire i costi associati alla terapia infusiva (Barton et al., 1998; Kokiotis, 2001). Uno studio americano ha evidenziato come il $77 \%$ degli infermieri sostiene di non eseguire un accertamento continuo e routinario del dispositivo venoso dopo la sua inserzione al fine di individuare precocemente le complicanze ad esso correlate (Kokiotis, 2001). Sono state sviluppate Linee Guida Pratiche e Standard Professionali per governare i problemi più rilevanti nella gestione degli accessi venosi, ma sono veramente molto scarse le pubblicazioni relative all'accertamento e alla scelta del dispositivo per gli accessi vascolari (Smith Higuchi et al., 2007). La linea guida per l'accertamento e la selezione del dispositivo per gli accessi vascolari sviluppata dall'associazione di infermieri Registered Nurses Association of Ontario (RNAO) (Registered Nurses Association of Ontario, 2004) è un punto di riferimento così come le indicazioni riportate dal Royal College of Nursing (Royal College of Nursing, 2010). L'aggiornamento, l'istruzione e la formazione specifica per la gestione degli accessi vascolari diviene per gli infermieri un passaggio fondamentale tanto che esso è riportato, insieme alla costituzione di un team specializzato, come raccomandazione in molte Linee Guida (Centers for Disease Control and Prevention, 2002; Registered Nurses Association of Ontario, 2004).

Nonostante gli infermieri dimostrino di avere buone conoscenze, non sempre le mettono in pratica (Karadeniz et al., 2003). Considerando l'alto rischio di complicanze correlate ai dispositivi venosi, è importante valutare l'aderenza alle raccomandazioni delle più recenti Linee Guida da parte degli operatori sanitari, in particolare dagli infermieri. La Linea Guida Assessment and Device Selection for Vascular Access (Registered Nurses Association of Ontario, 2004) raccomanda di eseguire un accertamento vascolare del paziente, selezionare il dispositivo più adatto, educare il paziente sul dispositivo, registrare le informazioni sul dispositivo, aggiornare gli infermieri sulla terapia infusiva, utilizzare infermieri esperti per ottenere esiti ottimali, organizzare un sistema di valutazione della qualità, educare ed informare pazienti e caregiver per la continuità assistenziale, applicare le Linee Guida nelle organizzazioni sanitarie.

Gli scopi di questo studio sono: indagare la presenza di una procedura per la scelta del dispositivo venoso più adeguato al paziente; descrivere i criteri utilizzati dagli infermieri per la scelta del dispositivo venoso più adeguato al paziente e per la valutazione del letto vascolare; descrivere se viene effettuata la valutazione del letto vascolare e quando essa viene eseguita.

\section{METODI}

Disegno di studio: descrittivo correlazionale.

Campione: tutti gli infermieri di almeno un reparto di ogni Dipartimento di un'Azienda Ospedaliera dell'Emilia Romagna. I reparti sono: Neurologia, Geriatria, Chirurgia Generale, Oncologia Day Hospital, Ematologia Bassa Carica Microbica, Ematologia Day Hospital, Medicina Generale, Lungodegenza, Radiologia, Pediatria, Cardiologia. Il campione è composto da 290 Infermieri.

Strumento raccolta dati: questionario anonimo costruito ad hoc e pre-testato. Il questionario è composto da 19 domande chiuse a risposta multipla e da 4 domande aperte (Allegato 1). I questionari somministrati, restituiti e ritenuti validi sono stati 227 .

Modalità di raccolta dati: il questionario è stato somministrato e raccolto da un infermiere del gruppo di ricerca che lo ha consegnato personalmente agli infermieri del campione all'inizio del turno di lavoro dal 3 all'11 Giugno 2010 e raccolto a fine turno.

Analisi dei dati: per le analisi dei dati sono stati utilizzati i software Microsoft Excel e EpiInfo. I test statistici utilizzati sono stati quelli relativi alla statistica descrittiva e il test Chi Quadrato (livello di significatività $=0.05)$.

\section{RISULTATI}

Gli infermieri partecipanti allo studio sono 290, i questionari restituiti e ritenuti validi 227 . Il campione è composto dall' $83 \%$ di femmine, il $50 \%$ possiede un Diploma di Infermiere Professionale il resto ha una formazione universitaria. Il $64 \%$ ha un'anzianità di servizio superiore ai 6 anni. Negli ultimi 5 anni il 55\% non ha partecipato a eventi formativi sugli accessi venosi e il 33\% non ha letto nessun articolo scientifico o linea guida sull'argomento. Le caratteristiche del campione sono illustrate nella Tabella 1. Il 98\% degli infermieri inserisce regolarmente nel corso del proprio lavoro dispositivi venosi, il $2 \%$ occasionalmente. I dispositivi venosi vengono utilizzati per infondere liquidi $(21 \%)$, farmaci $(24 \%)$, sostanze nutritive $(17 \%)$, sangue ed 


\begin{tabular}{|c|c|}
\hline caratteristiche & $\%$ \\
\hline \multicolumn{2}{|c|}{ Genere $(n=202)$} \\
\hline femmine & 83 \\
\hline maschi & 17 \\
\hline \multicolumn{2}{|c|}{ Tipo di Formazione $(n=213)$} \\
\hline Diploma Professionale & 50 \\
\hline Diploma Universitario & 14 \\
\hline Laurea & 34 \\
\hline Master di $1^{\circ}$ livello & 2 \\
\hline \multicolumn{2}{|c|}{ Anno conseguimento titolo $(n=205)$} \\
\hline $1975-1983$ & 10 \\
\hline 1984-1992 & 23 \\
\hline $1993-2001$ & 27 \\
\hline $2002-2010$ & 40 \\
\hline \multicolumn{2}{|c|}{ Anni di lavoro come infermiere $(n=216)$} \\
\hline$<1$ & 6 \\
\hline $1-3$ & 15 \\
\hline $4-6$ & 14 \\
\hline$>6$ & 64 \\
\hline \multicolumn{2}{|c|}{ Aggiornamenti sugli accessi venosi negli ultimi 5 anni $(n=212)$} \\
\hline Si & 45 \\
\hline No & 55 \\
\hline \multicolumn{2}{|c|}{ Lettura evidenze sugli accessi venosi negli ultimi 5 anni } \\
\hline Nessuna & 33 \\
\hline $1-3$ & 56 \\
\hline $4-6$ & 8 \\
\hline$>6$ & 3 \\
\hline
\end{tabular}

Tabella 1: caratteristiche degli infermieri appartenenti al campione.

\begin{tabular}{|c|c|c|c|}
\hline Momento di & $<6$ anni & $>6$ anni & totale \\
\hline stato vascolare & n [\%] & $\mathrm{n}[\%]$ & n [\%] \\
\hline All'ammissione & 27 [15] & $43[11]$ & $70[12]$ \\
\hline $\begin{array}{l}\text { Prima di inserire il } \\
\text { dispositivo }\end{array}$ & 42 [23] & $93[24]$ & $135[24]$ \\
\hline $\begin{array}{l}\text { Prima di riattivare il } \\
\text { dispositivo }\end{array}$ & 17 [9] & $43[11]$ & $60[11]$ \\
\hline Durante l'infusione & 17 [9] & $50[13]$ & 67 [12] \\
\hline $\begin{array}{l}\text { Quando compaiono } \\
\text { complicanze }\end{array}$ & 34 [18] & 60 [16] & 94 [16] \\
\hline $\begin{array}{l}\text { Quando non ci sono } \\
\text { più vene }\end{array}$ & 34 [18] & $57[15]$ & 91 [16] \\
\hline A intervalli regolari & $12[6]$ & 35 [9] & $47[8]$ \\
\hline Altro & $2[1]$ & $5[1]$ & $7[1]$ \\
\hline Totale & $185 *[100]$ & $386 *[100]$ & $571^{*}[100]$ \\
\hline
\end{tabular}

Tabella 2: quando gli infermieri con meno o più di 6 anni di servizio effettuano la valutazione dello stato vascolare. $*$ la somma assoluta eccede il numero degli infermieri del campione perché era possibile dare più di una risposta.

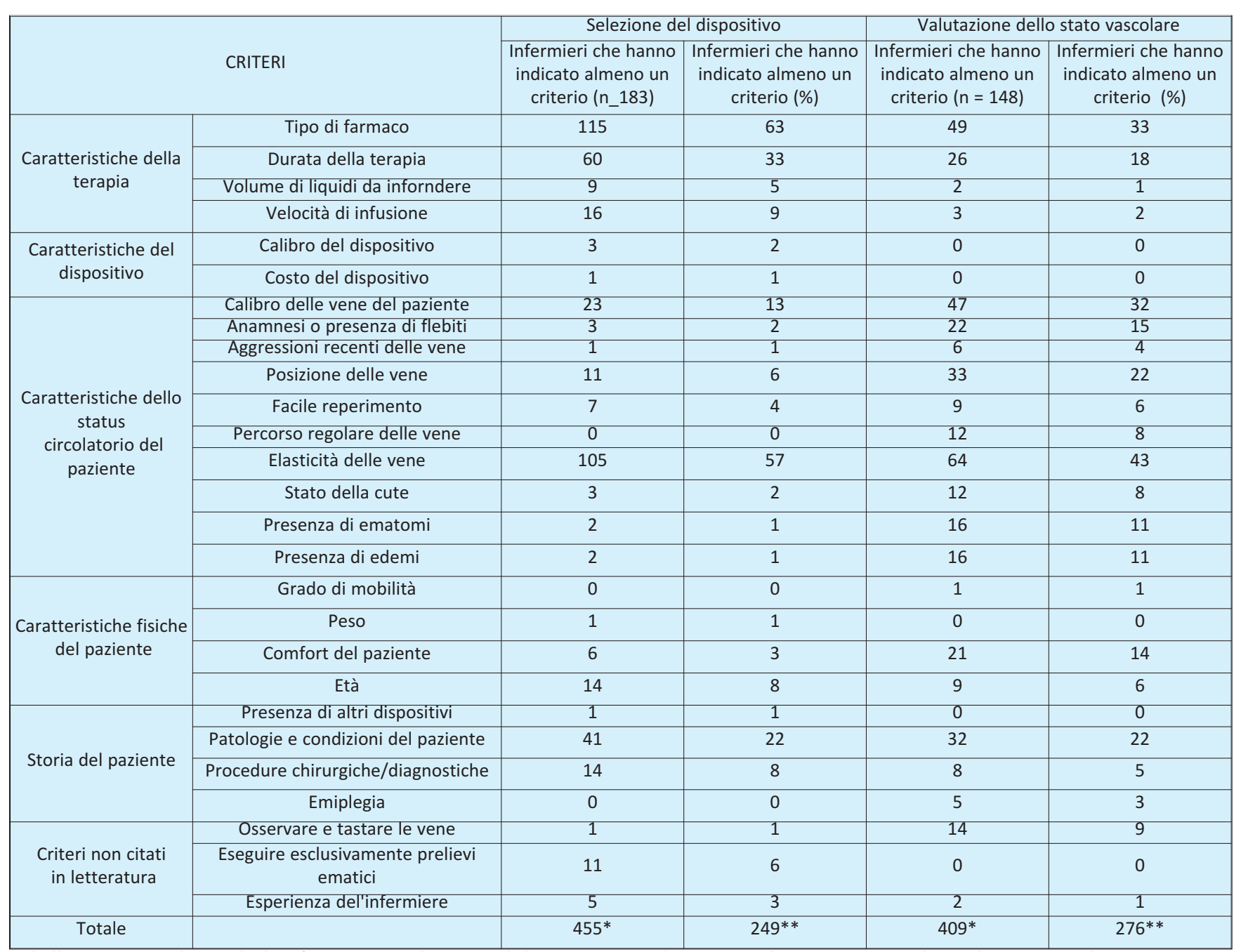

Tabella 3: criteri utilizzati dagli infermieri per selezionare il dispositivo più adeguato per il paziente e per valutare lo stato vascolare. * la somma assoluta eccede il numero di infermieri che hanno indicato il criterio perché era possibile dare più di una risposta. ** la somma delle percentuali eccede il $100 \%$ perché era possibile dare più di una risposta. 


\begin{tabular}{|c|c|c|c|c|c|c|c|c|c|c|}
\hline \multirow{4}{*}{ Criteri } & \multirow{3}{*}{$\begin{array}{c}\begin{array}{c}\text { Selezione del } \\
\text { dispositivo }\end{array} \\
\qquad n=420^{*}\end{array}$} & \multicolumn{4}{|c|}{ Differenze per anni di servizio: } & \multirow{4}{*}{$\begin{array}{c}\begin{array}{c}\text { Valutazione } \\
\text { dello stato } \\
\text { vascolare }\end{array} \\
\text { n }=409 * \\
\text { (n) }\end{array}$} & \multirow{2}{*}{\multicolumn{4}{|c|}{$\begin{array}{l}\text { Differenze per anni di servizio: } \\
\text { Valutazione dello stato vascolare }\end{array}$}} \\
\hline & & \multicolumn{4}{|c|}{ Selezione del dispositivo } & & & & & \\
\hline & & $<6$ anni & $>6$ anni & \multirow{2}{*}{$x^{2}$} & \multirow{2}{*}{$P=0.05$} & & $<6$ anni & $>6$ anni & \multirow{2}{*}{$x^{2}$} & \multirow{2}{*}{$P=0.05$} \\
\hline & (n) & $(\%)$ & (\%) & & & & (\%) & $(\%)$ & & \\
\hline $\begin{array}{c}\text { Caratteristiche della } \\
\text { terapia }\end{array}$ & 215 & 36 & 64 & 8 & 0.330759 & 80 & 19 & 81 & $1: 8$ & 0.04305555556 \\
\hline $\begin{array}{l}\text { Caratteristiche delle } \\
\text { vene }\end{array}$ & 131 & 45 & 55 & $11: 3$ & 0.046395 & 207 & 36 & 64 & $3: 4$ & 0.05833333333 \\
\hline Caratteristiche fisiche & 14 & 21 & 79 & $4: 1$ & 0.250761 & 66 & 32 & 68 & $2: 6$ & 0.04305555556 \\
\hline Storia del paziente & 55 & 46 & 54 & $3: 5$ & 0.323983 & 55 & 33 & 67 & $5: 6$ & 0.131496 \\
\hline $\begin{array}{l}\text { Caratteristiche } \\
\text { dell'infermiere }\end{array}$ & 5 & 0 & 100 & / & / & 1 & 0 & 100 & / & / \\
\hline
\end{tabular}

Tabella 4: criteri utilizzati per valutare lo stato vascolare e per selezionare il dispositivo

dagli infermieri con più di 6 anni di servizio e dagli infermiere con meno di 6 anni di servizio.

* la somma assoluta eccede il numero di infermieri perché era possibile dare più di una risposta.

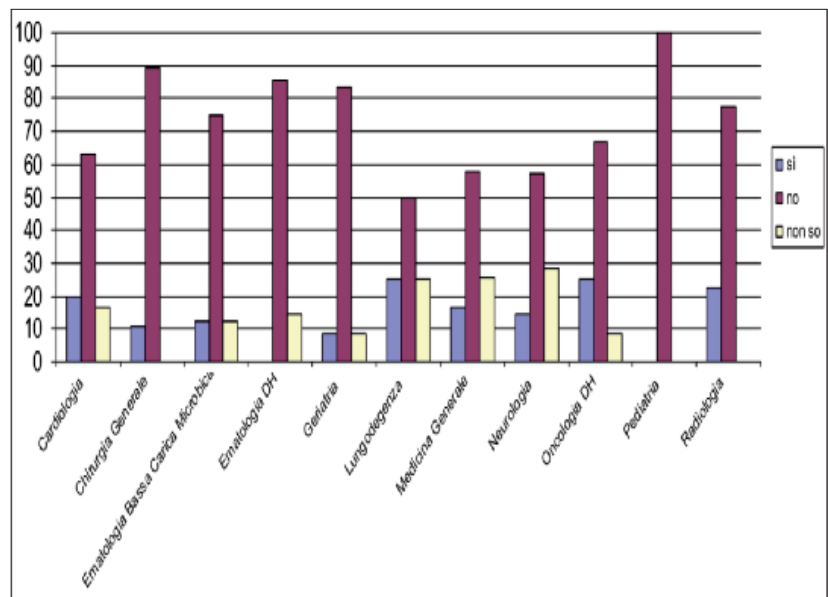

Grafico 1: presenza di una procedura per scegliere il dispositivo venoso più adatto per ogni paziente per Unità Operativa (in percentuale).

emoderivati (21\%), monitorare lo status emodinamico $(3 \%)$ e per eseguire prelievi ematici (14\%). I dispositivi utilizzati sono: ago butterfly (15\%), catetere periferico corto tipo intima (15\%), catetere periferico corto $(22 \%)$, catetere midline $(10 \%)$, catetere centrale a inserimento periferico (PICC) (2\%), catetere centrale percutaneo non tunnellizzato $(21 \%)$ e port-a-cath (15\%). Il 14\% degli infermieri dichiara che è in uso una procedura per scegliere il dispositivo venoso più adatto per ogni paziente, il $70 \%$ afferma che non è in uso e il $16 \%$ no lo sa. Solo gli infermieri di Pediatria e di Ematologia Day Hospital dichiarano che non c'è una procedura, in Radiologia e in Pediatria nessuno afferma che non sa se c'è una procedura mentre in tutte le altre Unità Operative sono presenti tutte le possibili risposte (Grafico 1).

I dati analizzati mostrano che la valutazione del letto venoso viene eseguita in momenti diversi (Tabella 2). Il $24 \%$ del campione effettua la valutazione prima di inserire il dispositivo, il $12 \%$ al momento dell'ammissione mentre solo l' $8 \%$ a intervalli regolari. Anche se non è del tutto corretto tenere a riferimento solamente il numero di anni di lavoro per definire esperto un infermiere, l'anzianità di servizio come infermiere è uno dei criteri di inclusione del campione in uno studio statunitense sull'esperienza relativa alla venipuntura (Larsen et al., 2010). Abbiamo, quindi, ritenuto che in relazione alle attività legate alla venipuntura e alla gestione dei relativi dispositivi, gli infermieri con più di 6 anni di servizio siano infermieri esperti con elevate conoscenze ed esperienza. Confrontando questi ultimi con quelli con meno anni di servizio abbiamo osservato che non vi sono differenze significative relative al momento in cui viene effettuata la valutazione del letto venoso $\left(X^{2}=5.5, p=0.598928\right)$.

Vi è una differenza statisticamente significativa per quanto riguarda i criteri utilizzati per valutare lo stato vascolare e quelli utilizzati per scegliere il dispositivo $\left(X^{2}=196.0, p=6.12^{(-29)}\right)$. I criteri utilizzati nelle due attività sono elencati nella Tabella 3 .

Relativamente ai criteri utilizzati per scegliere il dispositivo, l'unica differenza statisticamente significativa tra gli infermieri con più di 6 anni di servizio e quelli con meno anni riguarda il criterio "caratteristiche delle vene" (Tabella 4).

\section{DISCUSSIONE}

Gli infermieri si trovano a manipolare molti tipi di dispositivi venosi durante la loro attività quotidiana. Relativamente agli scopi dell'utilizzo dei dispositivi il quadro delineato dai dati analizzati sembra essere sovrapponibile a quello riportato dalla letteratura ad eccezione del monitoraggio dello status emodinamico che ha una bassa frequenza, attività svolta prevalentemente dagli infermieri che lavorano nelle terapie intensive ma che non sono incluse nel campione dello studio. L'86\% degli infermieri indagati dichiara che non è in uso o non sa se c'è una procedura per scegliere il dispositivo venoso più adeguato al paziente e per questo si può supporre che nei reparti del campione non è presente una procedura che sostenga l'infermiere nella 
scelta del dispositivo. Il fatto che il 16\% del campione non sa se c'è una procedura nella propria unità operativa non è comunque un dato da sottovalutare in relazione all'organizzazione del lavoro e in particolare all'efficacia dei sistemi/meccanismi operativi. Questa riflessione sembra essere supportata anche dalle risposte relative al momento in cui viene effettuata la valutazione del letto venoso (Tabella 2). Infatti, sembra che non vi sia un momento definito per effettuare la valutazione e sembra che essa non venga eseguita a scadenze regolari. Pare invece, che la valutazione venga eseguita principalmente al momento dell'inserimento o della riattivazione del dispositivo o quando compaiono complicanze $(16 \%)$ o non ci sono più vene $(16 \%)$. Questo è un dato che contrasta con ciò che è raccomandato in letteratura infatti, la valutazione del letto venoso dovrebbe essere effettuata al momento dell'ammissione del paziente e, successivamente, a intervalli regolari poiché essa impatta significativamente sugli indicatori economici, terapeutici e di soddisfazione (Registered Nurses Association of Ontario, 2004 ).

I criteri utilizzati dagli infermieri per scegliere il dispositivo più adeguato per il paziente sono in parte quelli elencati in letteratura: trattamento farmacologico prescritto, lunghezza del trattamento e valutazione dello stato fisico. In letteratura vengono riportati 3 criteri da prendere in considerazione quando si valuta il farmaco prescritto: il tipo di fluido, il pH e l'osmolarità. Nessun infermiere del campione ha riportato il $\mathrm{pH}$ e l'osmolarità probabilmente perché includevano questi criteri nel tipo di farmaco. A tale riguardo è importante, come raccomandato in letteratura (Stranz, 2002), una volta identificato il tipo di fluido determinare il $\mathrm{pH}$ e l'osmolarità poiché soluzioni con $\mathrm{pH}$ acido o alcalino causano un danno endoteliale e conseguentemente flebiti e formazione di trombi. Infatti, il rischio di trombosi chimica è il problema chiave alla base della scelta del posizionamento della punta del catetere e quindi della scelta del device più appropriato (Ryder, 1995). Riguardo la lunghezza del trattamento, solo una parte del campione ha individuato come criterio la durata della terapia ma non ha citato criteri temporali, ad esempio un cut-off in giorni o ore, mentre la letteratura raccomanda fortemente di utilizzare un dispositivo di medio/lungo termine per quei pazienti che necessitano di un trattamento infusivo che supera i 6 giorni (Registered Nurses Association of Ontario, 2004).

Oltre a ciò, i criteri appartenenti alla valutazione dello stato fisico non sono stati menzionati come ad esempio il livello di attività, stile di vita, obesità, stato di idratazione e presenza di patologia cronica. La presenza di una patologia cronica può impattare direttamente sulla scelta del dispositivo venoso soprattutto per preservare le vene che è uno degli obiettivi indicati in letteratura per i pazienti con malattie croniche che richiedono accessi vascolari a lungo termine (Bowen Santolucito, 2001). In effetti, il dispositivo vascolare centrale non è storicamente considerato fino a quando gli accessi periferici non sono esausti (Registered Nurses Association of Ontario, 2004).

Se comparati con la letteratura i criteri dichiarati dal campione per la scelta del dispositivo appaiono incompleti relativamente alle risorse e limitazioni dei pazienti (presenza di un caregiver a domicilio), alle caratteristiche dell'ambiente casalingo (la presenza di acqua sanitaria e servizi igienici, di elettricità e frigorifero, di uno spazio per stoccare le forniture, la presenza del telefono), all'abilità del paziente/caregiver di gestire il dispositivo e somministrare la terapia, alle risorse economiche. Bisogna però tenere conto che i pazienti trattati dal campione sono ospedalizzati e che, molto probabilmente, i dispositivi venosi sono rimossi prima della dimissione. Appaiono assolutamente secondari altri criteri come la storia clinica, la disponibilità del dispositivo e le preferenze del paziente.

Sebbene vi sia una differenza statisticamente significativa tra i criteri utilizzati dagli infermieri per effettuare una valutazione del letto venoso e quelli utilizzati per decidere quale dispositivo utilizzare $\left(X^{2}=196.0\right.$, $\left.\mathrm{p}=6.12^{(-29)}\right)$, molti criteri vengono utilizzati indifferentemente per entrambe le attività (Tabella 3). Nell'effettuare la valutazione dello stato vascolare dovrebbero essere tenuti in considerazione criteri riferibili alle caratteristiche dello status circolatorio del paziente (circolazione sanguigna, linfedema/edema, ematomi, tumefazioni post-operatorie, flebiti) e allo stato delle vene (elasticità, regolarità, calibro, posizione, percorso). Criteri quali il tipo di farmaco, la lunghezza temporale del trattamento, il livello di attività, non dovrebbero essere tenuti in considerazione quando si effettua un assessment dello stato vascolare. Probabilmente gli infermieri appartenenti al campione non hanno chiara la differenza tra la valutazione del letto venoso e la valutazione del dispositivo più adatto per il paziente. Quest'ultima non può solamente tenere in considerazione gli aspetti strettamente fisici ma, piuttosto, deve basarsi su aspetti riferibili ad una presa in carico globale, presa in carico che dovrebbe essere effettuata dal professionista infermiere il quale si occupa oltre che del to cure anche e soprattutto del to care.

\section{CONCLUSIONI}

Questo studio ha come obiettivi quelli di indagare la presenza di una procedura per la scelta del dispositivo venoso più adeguato al paziente, di descrivere $\mathrm{i}$ criteri utilizzati dagli infermieri per la scelta del dispositivo venoso più adeguato al paziente e per la valutazione del letto vascolare infine, di descrivere se viene 
effettuata la valutazione del letto vascolare e quando essa viene eseguita.

Nelle unità operative che hanno partecipato all'indagine non è in uso una procedura per la scelta del dispositivo più adeguato al paziente come raccomandato dalla letteratura. In letteratura si raccomanda l'utilizzo di strumenti, ad esempio algoritmi, per supportare l'infermiere nell'accertamento e nella selezione del dispositivo (Registered Nurses Association of Ontario, 2004; Galloway, 2002). I dati mostrano come vi sia poca chiarezza relativamente ai criteri utilizzati per scegliere il dispositivo e valutare lo stato del letto vascolare, oltre che una grande eterogeneità di comportamento relativa al momento in cui effettuare l'accertamento del letto vascolare.

\section{RILEVANZA PER LA PRATICA}

La gestione dei dispositivi venosi è un importante aspetto della pratica quotidiana degli infermieri, poiché essi sono sempre più frequentemente utilizzati per i trattamenti diagnostici e terapeutici dei pazienti. L'infermiere è il professionista sanitario che in prima persona si occupa della loro gestione e per questo è indispensabile che le sue scelte assistenziali si basino sulle migliori evidenze disponibili. Soltanto in questo modo egli sarà in grado di erogare un'assistenza efficace che permette di soddisfare le richieste dell'utenza attraverso la riduzione del discomfort e delle complicanze associate a questo tipo di dispositivi. È indispensabile che l'infermiere possegga le conoscenze necessarie a individuare il device più appropriato per il paziente e sappia gestirlo nel migliore dei modi. Attraverso un aggiornamento continuo su questo tema l'infermiere sarà in grado di avere conoscenze solide che permettano la presa di decisioni assistenziali adeguate alle richieste dell'utenza. Anche lo sviluppo di procedure che supportino le decisioni dell'equipe sanitaria in merito alla gestione dei dispositivi venosi, dalla scelta del più adeguato fino alla sua rimozione, può aumentare la qualità assistenziale erogata.

\section{BIBLIOGRAFIA}

Barton, A., Danek, G., Johns, P., \& Coons, M. (1998). Improving patient outcome through CQI: Vascular access planning. Journal of Nursing Care Quality, 13 (2), 77-85.

Bowen Santolucito, J. (2001). A retrospective evaluation of the timeliness of physician initiated PIC referrals. Journal of Vascular Access Devices, fall, 20-26.

Galloway, M. (2002). Using benchmarking data to determine vascular access device selection. Journal of
Infusion Nursing, 25 (5), 320-325.

Lundgren, A., \& Ek, A.C. (1996). Factors influencing nurses' handling and control of peripheral intravenous lines - an interview study. International Journal of Nursing Studies, 33, 131-42.

Kagel, E.M., \& Rayan, G.M. (2004). Intravenous catheter complications in the hand and forearm. The Journal of Trauma, 56 (1): 123-27.

Maki, D.G., Kluger, D.M., \& Crnich, C.J. (2006). The risk of bloodstream infection in adult with different intravascular device: a systematic review of 200 published prospective studies. Mayo Clinic Proceedings, 81 (9), 1159-71.

Tagalakis. V., Kahn, S.R., Libman, M., \& Blostein, M. (2002). The epidemiology of peripheral vein infusion thrombophlebitis: a critical review. American Journal of Medicine, 113 (2): 146-51.

Myrianthefs, P., Sifaki, M., Samara, I., \& Baltopoulos, G. (2005). The epidemiology of peripheral vein complication: avaluation of the efficiency of differing methods for the manteinance of catheter patency and thrombophlebitis prevention. Journal Evaluation of Clinical Practice, 11 (1): 85-89.

Maki, D.G., \& Ringer, M. (1987). Evaluation of dressing regimens for prevention of infection with peripheral intravenous catheters. Gauze, a transparent polyurethane dressing, and an iodophor-transparent dressing. JAMA, 258 (17), 2396-2403.

Smith, B. (2006). Peripheral intavenous catheter dwell times: a comparison of 3 securement methods for implementation of 96-hour scheduled ch'ange protocol. Journal of Infusion Nursing, 29(1), 14-17.

Kokiotis, K. (2001). New trends in vascular access therapy. Journal of Vascular Access Device, Summer, 7-17.

Smith Higuchi, K.A., Edwards, N., Danseco, E., Davies, B., \& McConnel, H. (2007). Development of an evaluation tool for clinical practice guideline on nursing assessment and device selection for vascular access. Journal of Infusion Nursing, 30 (1), 45-54.

Registered Nurses Association of Ontario (2004). Assessment and device selection for vascular access with 2008 supplement. Toronto, Ontario. Data accesso 14 maggio, 2012. da http://www.rnao.org/Page .asp?PageID=924\& ContentID=718

Royal College of Nursing (2010). Standards for infusion therapy. Third Edition, January 2010. London. Data accesso 14 maggio, 2012. da http://www.rcn.org.uk/_data/assets/pdf_file/0005/78 593/002179.pdf

Centers for Disease Control and Prevention (2002). Guidelines for the prevention of intravascular catheter-related infections. Morbidity and Mortality Weekly Report 51 (RR-10), 1-32. Data accesso 14 maggio, 2012. da http://www.cdc.gov/mmwr/indrr_ 2002.html

Karadeniz, G., Kutlu, N., Tatlisumak, E., \& Ozbakkaloglu, B. (2003). Nurses' knowledge regarding patients with intravenous catheter and phlebitis interventions. Journal of Vascular Nursing, 21 (2), 44-49. 
Larsen, P., Eldridge, D., Brinkley, J., Newton, D., Goff, D., Hartzog, T., Saad, N.D., \& Perkin, R. (2010). Pediatric peripheral intravenous access: does nursing experience and competence really make difference?. Journal of Infusion Nursing, 33 (4), 226-35.
Stranz, M. (2002). Adjusting pH and osmolarità levels to fit standards and practices. Journal of Vascular Access Devices, fall, 12-17.

Ryder, M. (1995). Peripheral access options. Surgical Oncology Clinics of North America, 4 (3), 395-427.
1.Nella tua struttura i dispositivi venosi vengono utilizzati per (è possibile dare più risposte):

口 Infondere fluidi

口 Infondere farmaci

a Infondere sostanze nutritive

口 Infondere sangue ed emoderivati

- Monitorare lo status emodinamico

Eseguire prelievi ematici

口 Altro (specificare)

2. Nella tua struttura quali tipi di dispositivi venosi vengono utilizzati (è possibile dare più risposte):

a Aghi farfalla

口 Aghi intima

口 Cateteri venosi periferici corti (CVP)

口 Cateteri venosi midline

a Cateteri venosi centrali periferici (PICC)

a Cateteri venosi centrali (CVC)

口 Port-a-cath

3. Nella tua Struttura viene eseguita una valutazione del letto venoso del paziente?

口 Sì $\square$ No Occasionalmente $\square$ Non so

4. Se sì, di norma quando viene eseguita la valutazione (è possibile dare più risposte):

口 Al momento dell'ammissione del paziente in Struttura

- Prima di inserire un dispositivo vascolare

口 Prima di riattivare il dispositivo vascolare

口 Durante l'infusione

Q Quando compaiono complicanze relative al dispositivo vascolare

口 Quando il paziente "non ha più vene"

口 A scadenze regolari

口 Altro (specificare)

5. Nella tua Struttura è in uso una procedura/protocollo per valutare il dispositivo venoso più adatto per ogni paziente?

$$
\text { ¿ì } \square \text { No } \square \text { Nonso }
$$

6. Se sì, quali criteri sono indicati nella procedura/protocollo per valutare il dispositivo venoso più adatto per ogni paziente?

$\overline{7 . \text { Se viene eseguita la valutazione per scegliere il dispositivo venoso più }}$ adatto per ogni paziente, quale professionista la esegue (è possibile dare più risposte)?

口 Infermiere

a Coordinatore Infermieristico

口 Medico

口 Infermiere e medico in collaborazione

口 Altro (specificare)

8. Se viene eseguita la valutazione, il professionista che la esegue ne registra l'esito?
口Sì
№
Occasionalmente

9. L'equipe infermieristica tiene in considerazione l'esito della valutazione per decidere quale dispositivo scegliere

$\square$ Sì

$\square$ No

Occasionalmente

口 Solo quando il paziente "non ha più vene"

10.L'equipe medica tiene in considerazione l'esito della valutazione per

decidere quale dispositivo venoso scegliere?

口 $\quad$ Sì

口 No

O Occasionalmente

- Solo quando il paziente "non ha più vene"
11. Nel corso del tuo lavoro ti capita di inserire un dispositivo venoso?

$$
\text { 口SI } \square \text { No } \square \text { Occasionalmente }
$$

12. Quali dispositivi venosi inserisci (è possibile dare più risposte)?

a Aghi farfalla

․ Aghi intima

C Cateteri venosi periferici corti (CVP)

- Cateteri venosi midline

a Cateteri venosi centrali periferici (PICC)

口 Cateteri venosi centrali (CVC)

a Port-a-cath (NO AGO GRIPPER)

13. Quali criteri utilizzi per decidere quale dispositivo venoso scegliere per il paziente?

14. Prima di inserire un qualsiasi dispositivo venoso esegui una valutazione del letto venoso del paziente?

$$
\text { पSì } \square \text { No } \square \text { Occasionalmente }
$$

15. Se sì, quali criteri di valutazione utilizzi?

16.Genere:
Maschio $\quad \square$ Femmina

17. Titolo di studio posseduto (indicare quello più elevato):

口 Diploma Regionale di Infermiere Professionale

口 Diploma Universitario per Infermiere

L Laurea in Infermieristica

- Master di primo livello (specificare):

- Laurea Magistrale in Scienze Infermieristiche/Ostetriche

- Master di secondo livello (specificare):

18. Anno di conseguimento del titolo di studio:

19. Da quanti anni ricopri il ruolo di Infermiere?

口 Da meno di un anno

口 Da 1 a 3 anni

- $\mathrm{Da} 4$ a 6 anni

口 Da più di 6 anni

20. Negli ultimi 5 anni hai partecipato ad un corso/aggiornamento sulla gestione degli accessi vascolari?

$$
\text { 口 }
$$

21. Negli ultimi 5 anni quanti articoli scientifici/linee guida/revisioni sugli accessi vascolari hai letto?

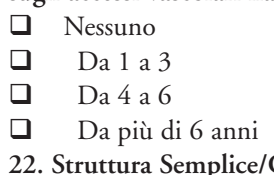

22. Struttura Semplice/Complessa di appartenenza:

a Cardiologia $\square$ Chirurgia 2

घ Ematologia BCM a Ematologia Day Hospital

G Geriatria a Lungodegenza

口 Medicina $\square$ Neurologia

口 Oncologia Day Hospital $\square$ Pediatria

口 Radiologia

23. Da quanti anni fai parte dell'equipe della tua Struttura?

口 Da meno di un anno

․ Da 1 a 3 anni

口 $\quad$ Da 4 a 6 anni

ㅁ Da più di 6 anni 